\title{
Learning from Aviation to Improve Safety in the Operating Room - a Systematic Literature Review
}

\author{
Linda S.G.L. Wauben",2,3*, Johan F. Lange, MD², Richard H.M. Goossens ${ }^{\mathbf{3 , 4}}$ \\ ${ }^{1}$ Department of BioMechanical Engineering, Faculty of Mechanical, Maritime and \\ Materials Engineering; ${ }^{3}$ Faculty of Industrial Design Engineering, Delft University of \\ Technology, Delft, The Netherlands \\ ${ }^{2}$ Department of Surgery; ${ }^{4}$ Department of Neuroscience, Erasmus University Medical \\ Center, Rotterdam, The Netherlands
}

Submitted December 2011. Accepted for publication March 2012.

\begin{abstract}
Lessons learned from other high-risk industries could improve patient safety in the operating room (OR). This review describes similarities and differences between high-risk industries and describes current methods and solutions within a system approach to reduce errors in the OR. PubMed and Scopus databases were systematically searched for relevant articles written in the English language published between 2000 and 2011. In total, 25 articles were included, all within the medical domain focusing on the comparison between surgery and aviation. In order to improve safety in the OR, multiple interventions have to be implemented. Additionally, the healthcare organization has to become a 'learning organization' and the OR team has to become a team with shared responsibilities and flat hierarchies. Interpersonal and technical skills can be trained by means of simulation and can be supported by implementing team briefings, debriefings and cross-checks. However, further development and research is needed to prove if these solutions are useful, practical, and actually increase safety.
\end{abstract}

Keywords: system approach, training, patient safety, aviation, error

\section{INTRODUCTION}

Extensive developments in surgery and in related disciplines have made the operating room (OR) environment and the procedures more complex [1-5]. This surgical complexity is defined by many factors, such as the patients and their condition, the procedure's complexity, the team's expertise, the equipment design and use, communication, workload, and the urgency and uncertainty of decisions [3-6]. Such increased complexity results in a high incidence of errors which are costly from human, economic, and societal viewpoints

*Corresponding Author: Linda S.G.L. Wauben, PhD, MSc. Delft University of Technology, Department of BioMechanical Engineering, Faculty of Mechanical, Maritime and Materials Engineering, Mekelweg 2, 2628 CD Delft, The Netherlands. Phone: 003115278 9315. Fax: 003115278 4717. E-mail: 1.s.g.l.wauben@tudelft.nl Other authors: r.h.m.goossens@tudelft.nl; j.lange@erasmusmc.nl. 
[6-8]. Furthermore, the society has become ever more critical on non-transparency with regard to errors and adverse events in healthcare.

Errors in the OR are concentration of contributing factors and seldom derive from a single case [9]. The resultant complication is specific to a particular type of surgical procedure, but its cause is not unique to the case, type of surgical procedure, or the team [10]. In order to prevent errors, first a definition of error has to be established, which also has to be familiarized by medical professionals. The most common definition of an error is defined by the Institute of Medicine (IOM) and states: 'failure of a planned action to be completed as intended or the use of a wrong plan to achieve an aim' [7]. Two types of error can be distinguished: active and latent errors (Figure 1) [6, 7, 9, 11, 12]. At the sharp end are active errors which are inflicted by humans (frontline operator). Latent errors are found in the structure and process. Both types of error could lead to near misses, no harm events or adverse events, with the latter having negative consequences for the patient $[6,7,9,12]$.

Several studies have shown that $30-50 \%$ of errors can be prevented $[3,7,14]$. Recent insight gained from other high-risk industries (high reliability organizations) has shown that besides lacking technical expertise (technical skills), non-technical skills (interpersonal skills) also cause errors [6, 7, 15-17]. The currently dominant 'person approach' in healthcare focuses on improving individual technical skills and decreasing human variability by means of disciplinary actions, retraining and further education $[9,11]$. On the other hand, the 'system approach' recognizes that humans are fallible and that factors within the system and immediate working environment can lead to errors, which are to be expected and are inevitable $[4,6-8,11,15,18]$. Adopting a system approach could improve the process of learning from errors, support identification of possible sources leading to these errors, and reduce the occurrence of preventable patient safety incidents [7, 11, 18, 19]. Building defenses by altering attitudes and modifying behavioral norms, improving quality of equipment or technology, training of professionals (both technical and nontechnical skills), and implementing protocols and safety reporting systems are some examples expected to reduce errors $[1-3,5,6,8,11,15,18]$.

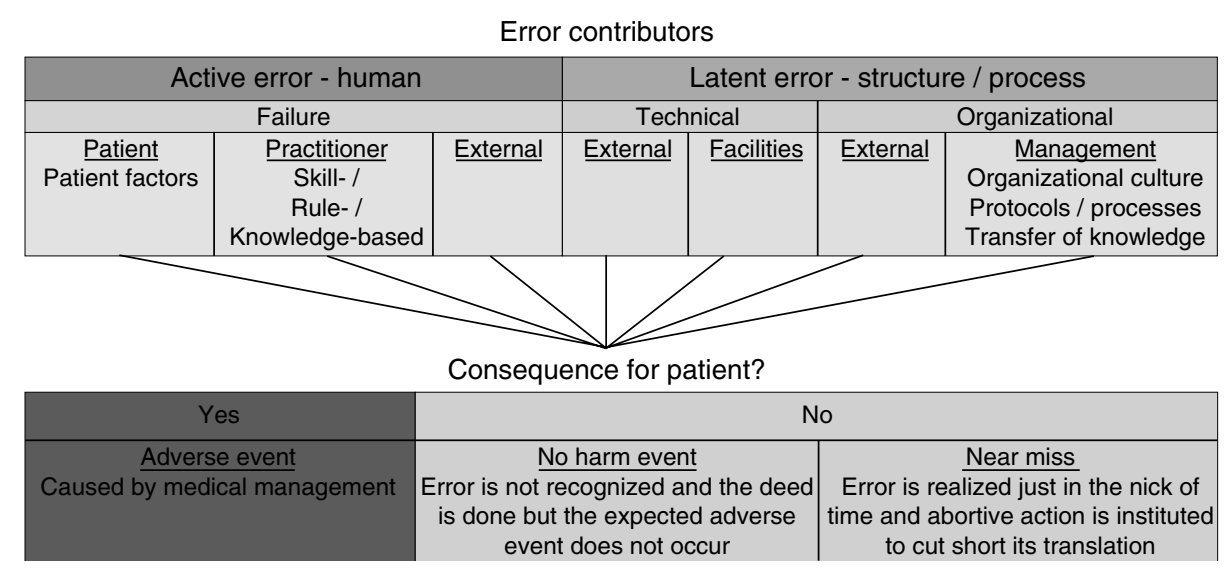

Figure 1. Types of error, error contributors and consequences for patients (based on $[6-9,12,13])$ 
Lessons learned from other high-risk industries could improve patient safety in the OR [7]. The objectives of this systematic literature review are (1) to compare and search for similarities and differences between the OR and other high-risk industries, and (2) to review current methods and solutions within a system approach employed in other high-risk industries and to evaluate if these solutions could be applicable to the OR to reduce errors.

\section{METHODS}

\subsection{Data Sources}

A systematic literature review was conducted using PubMed and Scopus databases as primary sources. The search was restricted to articles written in the English language published between January 2000 and November 2011. The search terms included in the first session were a combination of (a) 'system(s) approach' AND (b) 'surgery' or 'surgical procedure' or 'operating room' or 'operating theatre' or 'healthcare' AND (c) 'aviation' or 'oil industry' or 'nuclear industry' or 'high-risk industry' or 'process industry' or 'petrochemical industry' or 'offshore'. A second search session was carried out including the search terms: 'system(s) approach' AND 'patient safety' AND 'surgery', or 'surgical procedure' or 'operating room' or 'operating theatre'.

\subsection{Inclusion Criteria}

For this review, the articles should be original contributions including at least one of the following subjects: (a) similarities and differences between surgery and other highrisk industries, (b) elements within the system approach which influence safety in high-risk industries (also in the OR), and (c) solutions adopted from other high-risk industries to improve safety in the OR. Other inclusion criteria for articles were that subject of interest was the OR and the team members working in this environment, and that the article had to be published in a peer-reviewed journal or in a peer-reviewed conference proceeding.

\subsection{Exclusion Criteria}

Studies focusing on medication safety and other healthcare environments than the OR (e.g., emergency room, ward, intensive care unit) were excluded from this review. Furthermore, editorial letters, books, interviews, reviews, and comments/ perspectives were excluded as well.

\subsection{Data Extraction}

The first author retrieved each full-text article and systematically reviewed the articles based on the inclusion and exclusion criteria. Additionally, apparently relevant articles identified in the reference lists were hand-searched and retrieved as well.

\section{RESULTS}

In total, 25 articles were selected and included in this review: 12 articles via the first search session [1, 2, 13, 14, 20-27], four articles via the second search session [19, 28-30], and nine via the references $[10,11,16,17,31-35]$. All publications were 
within the medical domain and focused mainly on the comparison between surgery (the OR) and aviation.

\subsection{Similarities and Differences Between Surgery and Aviation}

In order to prevent errors in the OR, much can be learned from other high-risk industries, mainly from aviation [7]. Table 1 shows the similarities and differences between surgery and aviation, which were categorized into three groups.

Table 1. Similarities (comparable problems/conditions) and differences between surgery and aviation

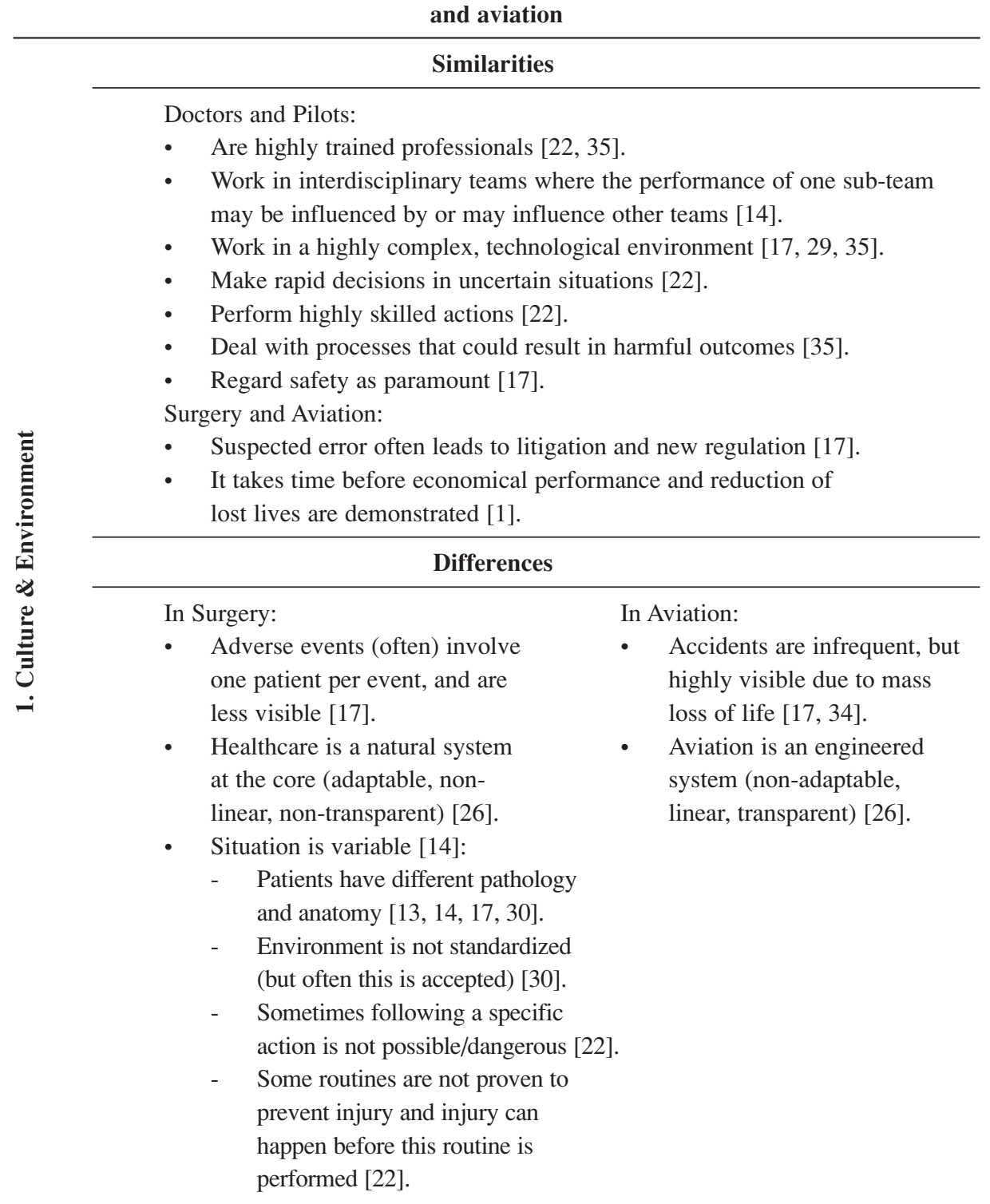


Table 1. Similarities (comparable problems/conditions) and differences between surgery and aviation (Continued)

\begin{tabular}{|c|c|c|}
\hline & $\begin{array}{l}\text { - } 82 \% \text { of surgeons and } 53 \% \text { of } \\
\text { anesthesiologists deny the effect } \\
\text { of personal stress on their } \\
\text { performance [16]. } \\
70 \% \text { of surgeons and } 47 \% \text { of } \\
\text { anesthesiologists deny the } \\
\text { effect of fatigue on critical } \\
\text { aspects of performance [16]. } \\
\text { - } 55 \% \text { of surgeons advocate } \\
\text { flat hierarchies [16]. } \\
\text { Surgeons are trained as } \\
\text { specialist [34]. } \\
\text { Surgeons are guided by } \\
\text { professional judgment first, then } \\
\text { by procedural control [35]. } \\
\text { Consequences of action } \\
\text { (consistent feedback) are not } \\
\text { immediately visible [34]. } \\
\text { Actions and decisions often } \\
\text { depend on subjective interpretation } \\
\text { of the surgeon [22]. } \\
\text { Is difficult to recognize failure } \\
\text { during or after surgery } \\
\text { (error-false-hypothesis or deadly } \\
\text { mindset) [22]. }\end{array}$ & $\begin{array}{l}\text { 53\% of pilots deny the } \\
\text { effect of personal stress } \\
\text { on their performance [16]. } \\
\text { - } \quad 26 \% \text { of pilots deny the } \\
\text { effect of fatigue on critical } \\
\text { aspects of performance } \\
{[16] .} \\
\text { - } 94 \% \text { of cockpit staff } \\
\text { advocate flat hierarchies [16]. } \\
\text { Pilots are trained as } \\
\text { generalist [34]. } \\
\text { Pilots are mainly guided } \\
\text { by procedure control [35]. } \\
\text { Rely on technology to provide } \\
\text { objective indicators [34]. }\end{array}$ \\
\hline \multicolumn{3}{|c|}{ Differences } \\
\hline 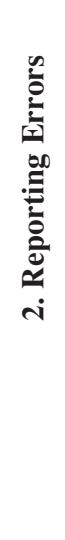 & $\begin{array}{l}\text { In Surgery: } \\
\text { Healthcare organizations lack } \\
\text { capacity for gathering the right } \\
\text { data and use non-standardized } \\
\text { error reporting which prohibits } \\
\text { processing the data into usable } \\
\text { information, and transforming } \\
\text { this information into knowledge } \\
\text { to improve safety }[17,20,25] \text {. }\end{array}$ & 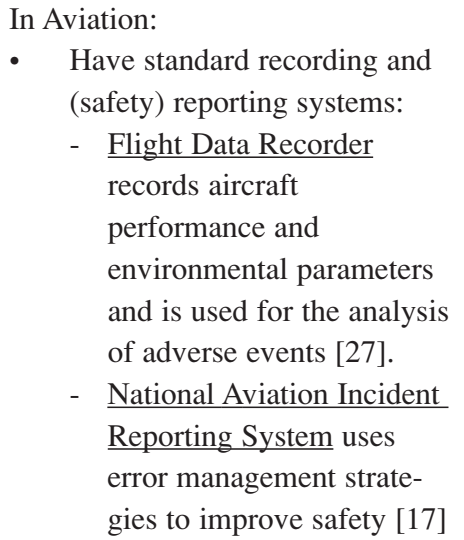 \\
\hline
\end{tabular}

Table 1. (Continued) 
Table 1. Similarities (comparable problems/conditions) and differences between surgery and aviation (Continued)

- Reporting safety concerns is not encouraged in surgery $[16,31]$.

- Underreporting of incidents and substantial pressures to cover up errors $(80 \%)$ caused by fear of blame, time pressure, resource constraints, perception that reporting is unnecessary, lack of clear definition $[16,20,25,31,33]$.

- Aviation Safety Information Analysis and Sharing system: Important safety information derived from the analyses by safety analyst is communicated to other airlines and private pilots and is used for training and learning [29, 34, 35].

- National Transportation Safety Board provides independent recommendations for safety [34, 35].

- Aviation Safety Information System monitors potential danger [35].

- Reporting safety concerns is encouraged and reporting incidents is voluntary [17, 34, 35].

- Deal with errors nonpunitively and proactive $[16,17,31,34,35]$.

\section{Similarities}

In Surgery and Aviation:

- $\quad 70-75 \%$ of errors are caused by lacking non-technical skills (e.g., lack of vigilance and failure to check, impaired decision making, absence of situation awareness, failure in interpersonal communication) [23].

\section{Differences}

In Surgery:

- Training is mainly focused on technical proficiency (e.g., operation time, motion analysis) [24].
In Aviation:

- Training includes nontechnical skills training by means of Crew Resource Management (CRM - 
Table 1. Similarities (comparable problems/conditions) and differences between surgery and aviation (Continued)

\begin{tabular}{cl}
\hline & $\begin{array}{l}\text { multidisciplinary team } \\
\text { training). CRM is part of }\end{array}$ \\
& education/training for 25 years \\
& {$[2,16,17,23,34]$.}
\end{tabular}

\subsubsection{Culture \& Environment}

Although the cockpit of an airplane and the OR are comparable in some aspects [1, $14,17,22,29,35]$, they are not similar. In contrast to aviation, adverse events in surgery involve one patient per event usually, which makes them less visible [17]. Other major differences exist in patient's variance and in the existing culture, mainly in acknowledging human error and human fallibility [1, 2, 13, 14, 16, 17, 22, 29, 30]. Surgical teams also have to work in a non-standardized environment [2, 17, 30, 35]. Furthermore, it is difficult to recognize failure during or after surgery as consequences of actions are not immediately visible due to lack of, e.g., predictive tools $[22,34]$.

\subsubsection{Reporting Errors}

Errors are inevitable and usually do not derive from carelessness of the actors as the system in which they work influences their actions. In order to understand the full cause of errors, they have to be analyzed (e.g., by means of systematic evaluation, root cause analysis), which requires reporting of errors. Compared to aviation, reporting errors is not encouraged in surgery $[16,17,31,34,35]$. On the contrary, there still exists substantial pressure to cover up errors, which makes errors underreported [16, 20, 25, 31, 33].

In surgery, safety-reporting systems are only recently implemented and used, which makes the learning and training effect limited up till now [17, 20, 25]. On the contrary, in aviation, recording and reporting errors is a common practice and is facilitated [17, $27,29,31,34,35]$.

\subsubsection{Team Skills and Training}

A similarity between surgery and aviation is that $70-75 \%$ or errors are caused by lacking non-technical skills [23]. In contrast to surgery, aviation started raising awareness for human error and multidisciplinary team training (Crew Resource Management, CRM) 25 years ago [2, 16, 17, 23, 34]. These training modules are certified, standardized, and use simulation to train team members [13]. 
Current medical training of surgeons is still mainly focused on technical proficiency, directed at the individual student or resident, and is often performed on the actual patient [24]. Non-technical skills are not systematically taught in the medical curriculum yet, but have to be acquired over time [23, 24].

In contrast to aviation, in surgery, it is still believed that individual surgeons cause surgical complications due to, for instance, errors in judgment, and inattention to detail, instead of these errors being (partly) caused by the system in which they have to work [21].

\subsection{Solutions to Prevent errors in Surgery}

The Institute of Medicine (IOM) report described that 'Much can be learned from the analysis of errors' [7]. The first steps are adopting a system approach and moving towards an open and non-punitive environment where human limitations are recognized and questioned. Furthermore, patient safety has to be the priority for the organization by providing resources [29]. Solutions from a system approach perspective can be categorized into four groups (see Table 2).

\subsubsection{Transparency \& Gaining Insight into the Process Leading to Errors}

In order to determine errors responsible for adverse events, near misses, and no harm events, an error classification system is needed [20, 21]. In order to understand the causes of errors, patient safety reporting systems should use combinations of methods, such as performance monitoring, observations, Healthcare Failure Mode and Effect Analysis, and Root Cause Analysis to obtain information of underlining contributors to errors $[10,11,17,19,21,25,29-31,33]$. These reporting systems should be easy-to-use and provide fast and effective feedback, consisting of corrective actions and addressing specific vulnerabilities in the care system, to raise awareness of safety issues [20, 21, 31, 33]. Starting a dialogue with OR team members that use local systems is important, as they must support the implementation of risk management systems. This is not done on a structural basis yet [20]. Adopting a proactive approach by sharing, training and learning from operational experience could lead to error prevention [20, 21, 25, 29]. Finally, technology, such as video and audio recording in the OR, improves the possibilities of objective assessment of skills $[11,22,27,35]$.

\subsubsection{Culture}

In order for the proposed solutions to work, an organizational safety culture has to be created $[11,17]$. This can be done by performing self-analysis of the organization and adopting a 'no blame and shame' policy for the actor of error where reporting errors has to be encouraged by engaging staff and providing incentives [16, 17, 20, 27, 29, 31, 33]. Healthcare professionals should be trained and educated on the basis of human factors principles [16, 17, 20, 22, 27, 29, 31]. Team members should also follow OR protocol and should not operate in an OR or use equipment that they cannot totally control [10, 17, 29-31, 33]. 
Table 2. Solutions to improve safety in the operating room

\begin{tabular}{|c|c|}
\hline & Solutions \\
\hline 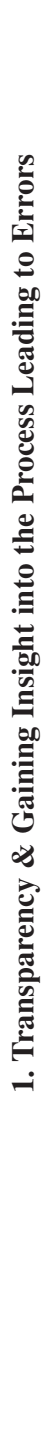 & 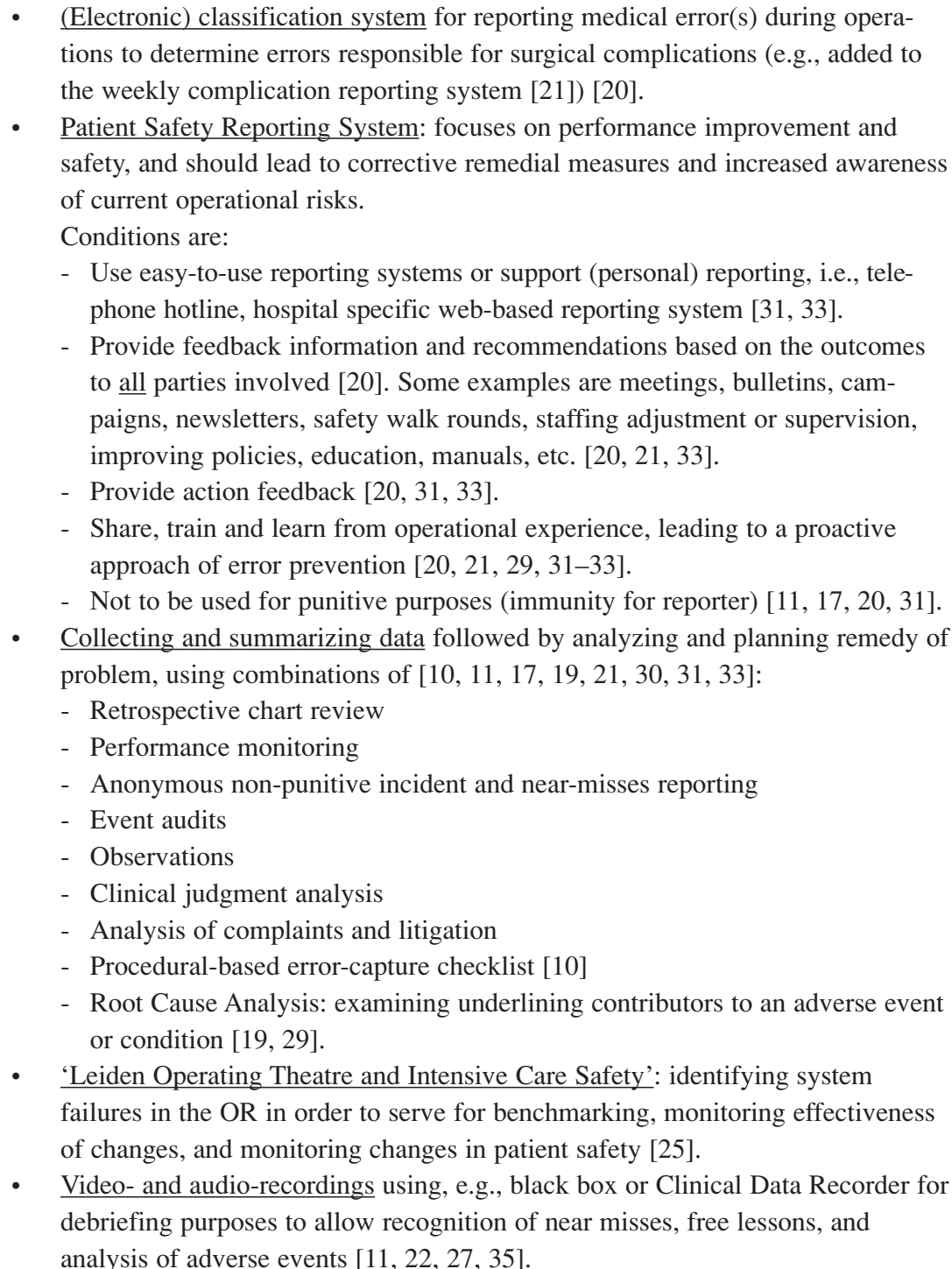 \\
\hline
\end{tabular}

Table 2. (Continued) 
Table 2. Solutions to improve safety in the operating room (Continued)

- Train basic Human Factors principles [22].

- No avoidance and denial of the fallibility of human performance [16, 17].

- Admit errors [16, 17, 20, 27, 31].

- No blame and shame for the actor of error (legal and ethical issues) [16, 17, 20, 27, 29, 31].

- Create an organizational safety culture [11, 17].

- Perform self-analysis of the organization [33].

- Encourage reporting errors by engaging staff and providing incentives [17, 29, $31,33]$.

- Do not operate in an environment or use equipment that the user cannot totally control [30].

- $\quad$ Follow protocol [10].

- Standardize surgical procedures [11].

- Standard terminology and classifications for, e.g., error and adverse event (better support to obtain valid and reliable data, and to assess the impact of a specific organizational intervention) [10, 20, 31, 32, 35].

- $\quad$ Standardize feedback from reporting systems to reporters and broad communities as a whole [20, 29].

- $\quad$ Standardize technology (quality and uniformity of instruments and equipment) $[11,35]$.

- $\quad$ Automate wisely: use technology to support the user [32].

- Reduce complexity and improve control of the entire OR environment (e.g., door movements, noise, phone calls) [11, 28, 32].

- Use checklists (paper, electronic, or computer-based) to reduce reliance on memory of normal, non-normal and emergency procedures for [1, 11, 29, 32]:

- Key points in the operation, cross-checking with the whole team [1, 22, 30, 32]

- Pre-operative briefings (improving safety attitude and double checking important patient and procedure related items) $[1,11]$

- Anesthesia equipment $[1,11]$

- Laparoscopic instruments [1,28]

- Use valid and reliable assessment systems on clinically relevant outcomes [2, 14]:

- Assessment of postoperative complications

- Cancellations of cases

- Delays in lists

- Returns to OR

- Recalls of surgeons or anesthesiologists to recovery

- Clinical incidents

- Use valid and reliable assessment systems for measuring both technical and non technical skills on clinically relevant outcomes $[2,14]$ :

Technical performance:

- Observation Clinical Human Reliability Assessment (OCHRA) [14]

Non-technical performance [2, 14, 19]: 
Table 2. Solutions to improve safety in the operating room (Continued)

\begin{tabular}{|c|c|}
\hline & $\begin{array}{l}\text { - Surgical Non Technical performance (NOTECHS) } \\
\text { - } \text { Anesthetists Non-Technical Skills assessments (ANTS) } \\
\text { - Situation Awareness Rating Technique (SART) } \\
\text { - } \text { Situation Awareness Global Assessment Technique (SAGAT) } \\
\text { - Observational Teamwork Assessment for Surgery (OTAS) } \\
\text { - Judgment Analysis }\end{array}$ \\
\hline 粶 & 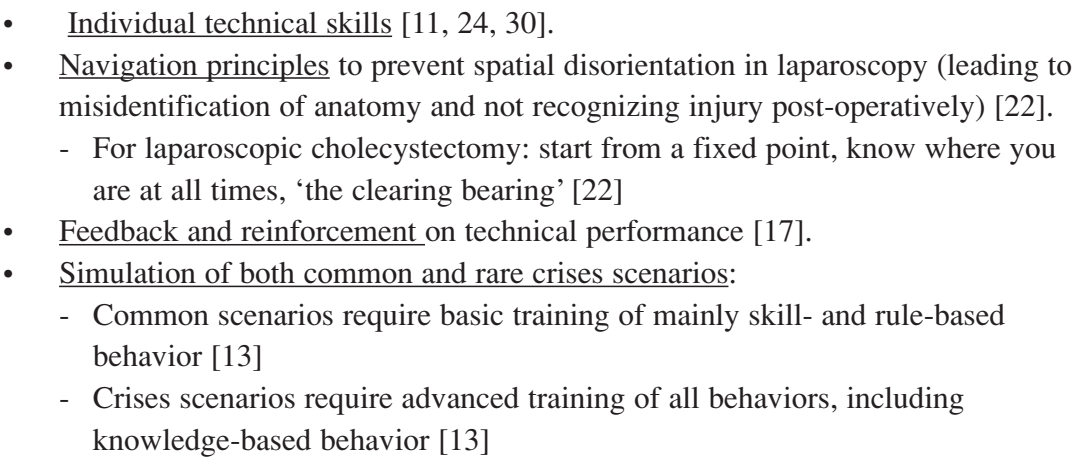 \\
\hline 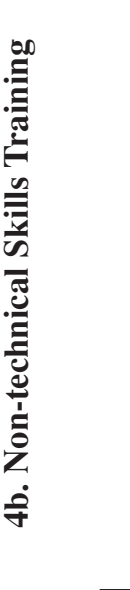 & $\begin{array}{l}\text { CRM, Team training, Cognitive competence training } \\
\text { Training aspects: }[2,10,14,17,23,29,30] \\
\text { - } \text { Situation awareness and vigilance (by controlling external distractions, antici- } \\
\text { pation of future events, appropriate use of all members of the team) } \\
\text { - } \text { Leadership and management } \\
\text { - } \text { Teamwork and cooperation } \\
\text { - Problem solving and decision making } \\
\text { - Communication processes } \\
\text { Attitudes: } \\
\text { - Assertiveness, inviting input, horizontal authority, flat hierarchy [16, 17, 22, 29] } \\
\text { - Recognizing effect of self fatigue, time pressure and personal worries [16, 22] } \\
\text { - Cross-checks [17, 22] } \\
\text { - Safety issues [10,11, 17] } \\
\text { Feedback and reinforcement on interpersonal performance [17] }\end{array}$ \\
\hline 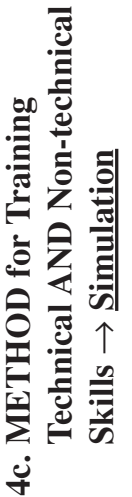 & $\begin{array}{l}\text { - Interdisciplinary simulations in an operational environment [19], e.g., the } \\
\text { 'simulated operating theatre' [23]. } \\
\text { Training of: } \\
\text { - Common and rare crises scenarios [24] } \\
\text { - Individual technical skills }[19,23,24] \\
\text { - Team skills }[19,23,24]\end{array}$ \\
\hline
\end{tabular}




\subsubsection{Standardization}

Besides standardization of definitions (terminology) and the interventions described above, the feedback from reporting systems to reporters and broad communities as a whole has to be standardized [20,29]. Surgical procedures and its technology can also be standardized [10, 11, 20, 31, 32, 35]. Moreover, the complexity of the entire OR environment can be reduced and the control improved (e.g., door movements, noise, phone calls) [11, 28, 32]. Additionally, checklists, briefings and debriefings can be used to create and maintain a safety culture in the OR [1, 11, 22, 28-30, 32]. Different designs ('call-do-response' or 'do-verify' method) can be employed for systematically checking important patient and procedural factors, involving all team members, and checking, e.g., the presence of equipment [1]. Furthermore, valid and reliable assessment systems are needed to assess the influence of both technical and nontechnical skills on clinical outcomes [2,14]. Currently, several assessment systems are used in surgery $[2,14,19]$.

\subsubsection{Training}

Training is needed to bridge the gap in skills and knowledge between residents and surgeons. Besides training individual technical skills (e.g., navigation principles, dexterity, hand-eye coordination), non-technical skills and focusing on safety issues, cognitive competence and behavior have to be trained as well $[2,10,11,14,16,17$, 22-24, 29, 30]. Team training depends on mentoring, culture, personality, feedback and reinforcement on interpersonal performance, and exposure to positive role models. Acceptance of non-technical skills training in healthcare might be slow, as this was also the case for CRM training in aviation, where acceptance was slow but steady.

Technical and non-technical skills can be trained by means of simulation. This has some advantages, such as inflicting no harm to the patient, teaching trainees the skills to control crisis situations (e.g., bleeding), teaching consequences of unsafe or inappropriate actions, providing objective feedback on technical and non-technical performance during 'real lifelike' procedures, and identifying trainees competences, or that trainees need further training to meet professional standards [13, 17, 19, 23, 24].

\section{DISCUSSION}

Improving patient safety in the OR has gained increased awareness over last decade. However, much has to be done to reach the same safety levels as, for instance, commercial aviation where the safety risk is $10^{-6}$ versus $10^{-3}$ for surgery [36]. Besides the solutions described above, healthcare organizations have to stress the importance of shared or organizational learning from process failure, in order to become a 'learning organization' [17, 20, 31, 37, 38]. A learning organization is 'one that is successful at acquiring, cultivating, and applying knowledge that can be used to help it continually adapt to change' and has "created the environment and culture for quality and safety, which foster team building, innovation, learning, and, most of all, open communication throughout the organization" [15]. This requires an environment of psychological safety in which team members can speak up and report errors without being blamed or data being used for 
medical malpractice legal cases $[5,6,11,17,38]$. It also requires leadership that propagates a persuasive vision to motivate and communicate urgency for change [38].

To improve safety, multiple interventions have to be implemented. These interventions should not work against each other and should be proportional to their impact on outcome (e.g., reduction of lost lives) and the cost of preventing them in both the short and long term [1]. Implementation requires commitment and participation of front-line team members to adapt the interventions to the context in which they operate $[1,6,17,20,34,35,38]$. Furthermore, the implementation method and the research design to study the effect and impact of the implemented interventions are also important. As errors do not always result in harm to patients, merely collecting mortality and morbidity rates is insufficient. Also the effect of system improvements is not always portrayed in these data. Therefore, additional research methods such as direct observations are needed $[5,6]$.

The solutions summarized in this review (Table 2) do not include all relevant factors in the system. For instance, Carayon et al. [39] show in their SEIPS model (Systems Engineering Initiative for Patient Safety) that the work system entails five components that are interrelated. However, this review's solutions mainly focus on the components 'Technology and tools', 'Organization' and 'Person', underreporting the components 'Tasks' and 'Environment'. Compared to the framework by Vincent et al. [5], the factor types 'Institutional context', 'Work environment', 'Task' and 'Patient' factors are missing. This could be explained by the wide scope of the present review, which limited going into details of various solutions. The solutions surveyed also need further development in order to be implemented. Some examples are the improvements of (1) high fidelity surgical simulators comparable to those used in anesthesia for knowledge-based training (which, e.g., requires knowledge of the behavior of soft tissue organs) [13, 23, 37], (2) valid reporting systems, including multiple modes of feedback, i.e., information and action feedback [20,31], and (3) objective recording systems in the OR to obtain insight into the causes of errors. (For research purposes, these systems already exist, e.g., Clinical Data Recorder [5, 6, 23, 27, 37]). Also, training objectives, needs and means for surgical training need to be defined [13].

This review is also limited by focusing on solutions within a system approach. While 'system(s) approach' is a familiar term in the healthcare research domain, and was reported in the IOM report 'To Err is Human', the authors found that this term has limited adoption in OR and surgical literature, as relatively limited articles were found. This probably explains why only few articles explicitly studying the use of checklists, briefings and debriefing were found $[1,11]$ in contrast to the many checklists used in the OR today to, e.g., support briefings and debriefings, check the presence and functioning of equipment and instruments, and support team interaction $[1,6,22,30$, 40-45]. Another way to improve safety during surgery is to perform collaborative cross-checks (double-checks, or the two-challenge rule) [37, 46-48]. Cross-checks are performed by at least two people with different perspectives who examine each others' actions and/or observable behavior to assess its validity and accuracy [47]. In the short term, they detect an erroneous assessment or action and improve coordination. [47]. Diamond and Mole [46] reported that cross-checking reduced perceptual errors and led 
to no biliary injury during laparoscopic gallbladder procedures. It also provides a structure for trainees to more readily recognize and respond to error prone situations and to 'speak up' to authority gradients [48]. In the long-term, cross-checking can also increase system resilience [47].

Another limitation was that only articles published in peer-reviewed journals and conference proceedings were included, which means that publications from regulatory institutions were outside the scope of this review. However, institutions such as the National Transportation and Safety Board, the Federal Aviation Administration, UK Civil Aviation Authority, and the European Aviation Safety Agency offer valuable information and insights that could be translated to improve safety in the OR [49-52]. They provide rules and regulation which, for instance, express the need to test technology 'before-the-job' (by means of, e.g., usability testing, validation testing, simulation-based testing), the need to train staff to gain equipment-specific knowledge and proficiency to use and maintain the technology, and the need to capture maintenance and operational information in order to assess the reliability.

Further research is needed to prove the effectiveness of safety reporting systems, training, and checklists to see whether these systems from other industries are useful and practical, and actually increase safety in the OR [1, 2, 15, 20, 23, 24, 29]. Finally, in order to improve patient safety, the gap between research findings, guideline development and their implementation in the OR has to be bridged [3].

\section{CONCLUSION}

High-risk industries, particularly aviation, have a protracted affair of improving safety by looking at the entire system, which can serve as input and inspiration for the OR. Nevertheless, adoption of a system approach in the OR is still relatively new. In order to improve safety in the OR, healthcare organizations have to become 'learning organizations'. This requires strong (horizontal) leadership communicating urgency for change and creating a safe culture to speak up and report error. Only then the processes leading to errors can be studied and future errors been reduced. The OR team has to become a team with shared responsibilities and flat hierarchies. Both interpersonal and technical skills can be trained by means of simulation and can be supported by implementing team briefings, debriefings and cross-checks.

To determine whether implementation of these multiple interventions is useful, practical, and actually increase safety, further development and research are needed. High fidelity surgical simulators (to train technical and non-technical skills) and valid reporting and recording systems have to be optimized, and training objectives, needs and means have to be defined. Finally, error proof procedures (checks, briefings, debriefings and cross-checks) have to be implemented and adopted by the OR teams.

\section{CONFLICT OF INTEREST}

The authors indicated no potential conflicts of interest. 


\section{REFERENCES}

[1] Verdaasdonk EG, Stassen LP, Widhiasmara PP and Dankelman J. Requirements for the design and implementation of checklists for surgical processes. Surg Endosc. 2009, 23(4):715-726.

[2] Sevdalis N, Davis R, Koutantji M, Undre S, Darzi A and Vincent CA. Reliability of a revised NOTECHS scale for use in surgical teams. Am J Surg. 2008, 196(2):184-190.

[3] Olsen S, Undre S and Vincent C. Safety in surgery: First steps towards a systems approach. Clinical Risk. 2005, 11(5):190-194.

[4] Undre S, Arora S and Sevdalis N. Surgical performance, human error and patient safety in urological surgery. British Journal of Medical and Surgical Urology. 2009, 2(1):2-10.

[5] Vincent C, Moorthy K, Sarker SK, Chang A and Darzi AW. Systems approaches to surgical quality and safety: from concept to measurement. Ann Surg. 2004, 239(4):475-482.

[6] Calland JF, Guerlain S, Adams RB, Tribble CG, Foley E and Chekan EG. A systems approach to surgical safety. Surg Endosc. 2002, 16(6):1005-1014; discussion 1015.

[7] Kohn LT, Corrigan JM and Donaldson MS. To Err is Human: Building a safer health system, National Academy Press, Washington DC, 2000.

[8] La Pietra L, Calligaris L, Molendini L, Quattrin R and Brusaferro S. Medical errors and clinical risk management: state of the art. Acta Otorhinolaryngol. Ital. 2005, 25(6):339-346.

[9] Reason J. Human error: models and management. BMJ. 2000, 320(7237):768-770.

[10] Catchpole KR, Giddings AE, de Leval MR, Peek GJ, Godden PJ, Utley M, Gallivan S, Hirst G and Dale T. Identification of systems failures in successful paediatric cardiac surgery. Ergonomics. 2006, 49(5-6):567-588.

[11] Dankelman J and Grimbergen CA. Systems approach to reduce errors in surgery. Surg Endosc. 2005, 19(8):1017-1021.

[12] Cuschieri A. Nature of Human Error. Implications for Surgical Practice. Ann. Surg. 2006, 244(5):642-648.

[13] Wentink M, Stassen LPS, Alwayn I, Hosman RJAW and Stassen HG. Rasmussen's model of human behavior in laparoscopy training. Surgical Endoscopy. 2003, 17(-):1241-1246.

[14] Mishra A, Catchpole K, Dale T and McCulloch P. The influence of non-technical performance on technical outcome in laparoscopic cholecystectomy. Surg Endosc. 2008, 22(1):68-73.

[15] Dotan BD. Patient safety organizations: A new paradigm in quality management and communication systems in healthcare. J. Clin. Eng. 2009, 34(3):142-146

[16] Sexton JB, Thomas EJ and Helmreich RL. Error, stress, and teamwork in medicine and aviation: cross sectional surveys. BMJ. 2000, 320(7237):745-749.

[17] Helmreich RL. On error management: lessons from aviation. BMJ. 2000, 320(7237):781-785.

[18] Aggarwal R, Undre S, Moorthy K, Vincent C and Darzi A. The simulated operating theatre: comprehensive training for surgical teams. Quality \& safety in health care. 2004, 13 (Suppl 1):i27-32.

[19] Arora S and Sevdalis N. Systems Approach to daily clinical care. Int J Surg. 2010, 8(2):164-166.

[20] Benn J, Koutantji M, Wallace L, Spurgeon P, Rejman M, Healey A and Vincent C. Feedback from incident reporting: information and action to improve patient safety. Quality \& safety in health care. 2009, 18(1):11-21.

[21] Fabri PJ and Zayas-Castro JL. Human error, not communication and systems, underlies surgical complications. Surgery. 2008, 144(4):557-563.

[22] Hugh TB. New strategies to prevent laparoscopic bile duct injury—surgeons can learn from pilots. Surgery. 2002, 132(5):826-835.

[23] Moorthy K, Munz Y, Adams S, Pandey V and Darzi A. A human factors analysis of technical and team skills among surgical trainees during procedural simulations in a simulated operating theatre. Ann Surg. 2005, 242(5):631-639. 
[24] Moorthy K, Munz Y, Forrest D, Pandey V, Undre S, Vincent C and Darzi A. Surgical crisis management skills training and assessment: a simulation-based approach to enhancing operating room performance. Ann Surg. 2006, 244(1):139-147.

[25] van Beuzekom M, Akerboom SP and Boer F. Assessing system failures in operating rooms and intensive care units. Quality \& safety in health care. 2007, 16(1):45-50.

[26] Drews FA and Fawcett D. Why healthcare is not like aviation: Control of natural and technical systems. Conf Proc 54th Human Factors and Ergonomics Society Annual Meeting 2010, HFES 2010. Year; 1; 369-373.

[27] Nascimento LN and Calil SJ. The clinical data recorder: What shall be monitored? Conf Proc 12th Mediterranean Conference on Medical and Biological Engineering and Computing, MEDICON 2010. Year; 29; 995-998.

[28] Rodrigues SP, Wever AM, Dankelman J and Jansen FW. Risk factors in patient safety: minimally invasive surgery versus conventional surgery. Surg Endosc. 2011.

[29] Thiagarajan RR, Bird GL, Harrington K, Charpie JR, Ohye RC, Steven JM, Epstein M and Laussen PC. Improving safety for children with cardiac disease. Cardiol. Young. 2007, 17(Suppl 2):127-132.

[30] Lien HH, Huang CC, Liu JS, Shi MY, Chen DF, Wang NY, Tai FC and Huang CS. System approach to prevent common bile duct injury and enhance performance of laparoscopic cholecystectomy. Surg Laparosc Endosc Percutan Tech. 2007, 17(3):164-170.

[31] Wilf-Miron R, Lewenhoff I, Benyamini Z and Aviram A. From aviation to medicine: applying concepts of aviation safety to risk management in ambulatory care. Quality \& safety in health care. 2003, 12(1):35-39.

[32] Nolan TW. System changes to improve patient safety. BMJ. 2000, 320(7237):771-773.

[33] Joshi MS, Anderson JF and Marwaha S. A systems approach to improving error reporting. J. Healthc. Inf. Manag. 2002, 16(1):40-45.

[34] Durso FT and Drews FA. Health care, aviation, and ecosystems: A socio-natural systems perspective. Current Directions in Psychological Science. 2010, 19(2):71-75.

[35] Tamuz M and Thomas EJ. Classifying and interpreting threats to patient safety in hospitals: Insights from aviation. Journal of Organizational Behavior. 2006, 27(7):919-940.

[36] Amalberti R, Auroy Y, Berwick D and Barach P. Five system barriers to achieving ultrasafe health care. Annals of internal medicine. 2005, 142(9):756-764.

[37] Muller M. Safety lessons taken from the airlines. Br J Surg. 2004, 91(4):393-394.

[38] Edmondson AC. Learning from failure in health care: frequent opportunities, pervasive barriers. Quality \& safety in health care. 2004, 13 Suppl 2(ii3-9.

[39] Carayon P, Schoofs Hundt A, Karsh BT, Gurses AP, Alvarado CJ, Smith M and Flatley Brennan P. Work system design for patient safety: the SEIPS model. Quality \& safety in health care. 2006, 15 Suppl 1(i50-58.

[40] Haynes AB, Weiser TG, Berry WR, Lipsitz SR, Breizat AH, Dellinger EP, Herbosa T, Joseph S, Kibatala PL, Lapitan MC, Merry AF, Moorthy K, Reznick RK, Taylor B and Gawande AA. A surgical safety checklist to reduce morbidity and mortality in a global population. N. Engl. J. Med. 2009, 360(5):491-499.

[41] Dekker-van Doorn CM, Wauben LSGL, Bonke B, Kazemier G, Klein J, Balvert B, Vrouenraets B, Huijsman R and Lange JF. Introducing TOPplus in the Operating Theatre, in: Flin R, Mitchell L (ed). Safer Surgery-Analysing behaviour in the operating theatre, Ashgate, Farnham, 2009, 151-171.

[42] World Health Organization (2008) WHO Guidelines for Safe Surgery (first edition).

[43] Verdaasdonk EG, Stassen LP, Hoffmann WF, van der Elst M and Dankelman J. Can a structured checklist prevent problems with laparoscopic equipment? Surg Endosc. 2008, 22(10):2238-2243.

[44] Buzink SN, van Lier L, de Hingh IH and Jakimowicz JJ. Risk-sensitive events during laparoscopic cholecystectomy: the influence of the integrated operating room and a preoperative checklist tool. Surg Endosc. 2010, 24(8):1990-1995. 
[45] de Vries EN, Prins HA, Crolla RM, den Outer AJ, van Andel G, van Helden SH, Schlack WS, van Putten MA, Gouma DJ, Dijkgraaf MG, Smorenburg SM and Boermeester MA. Effect of a comprehensive surgical safety system on patient outcomes. N. Engl. J. Med. 2010, 363(20):1928-1937.

[46] Diamond T and Mole DJ. Anatomical orientation and cross-checking-the key to safer laparoscopic cholecystectomy. Br J Surg. 2005, 92(6):663-664.

[47] Patterson ES, Woods DD, Cook RI and Render ML. Collaborative cross-checking to enhance resilience. Cognition, Technology \& Work. 2007, 9(3):155-162.

[48] Pian-Smith MC, Simon R, Minehart RD, Podraza M, Rudolph J, Walzer T and Raemer D. Teaching residents the two-challenge rule: a simulation-based approach to improve education and patient safety. Simul Healthc. 2009, 4(2):84-91.

[49] Federal Aviation Administration. http://rgl.faa.gov. Accessed May 8, 2012.

[50] UK Civil Aviation Authority. http://www.caa.co.uk. Accessed May 8, 2012.

[51] National Transportation Safety Board. Introduction of Glass Cockpit Avionics into Light Aircraft. 2010. http://www.ntsb.gov/safety/safetystudies/SS1001.html. Accessed May 8, 2012.

[52] European Aviation Safety Agency. http://www.easa.europa.eu. Accessed May 8, 2012. 



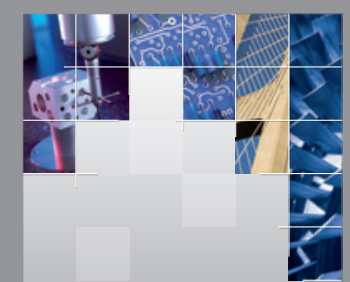

\section{Enfincering}
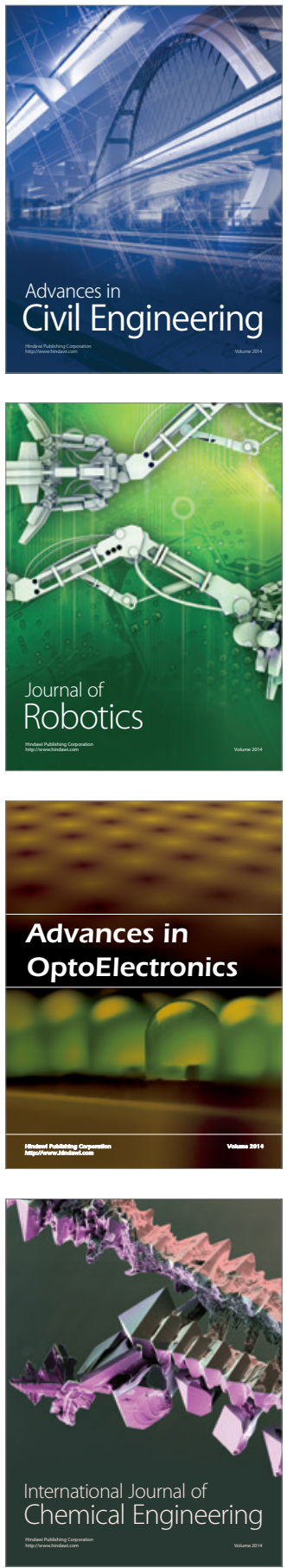

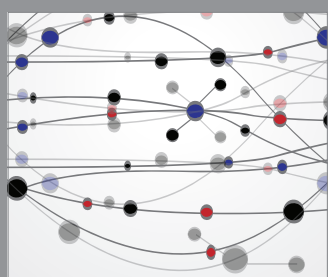

The Scientific World Journal

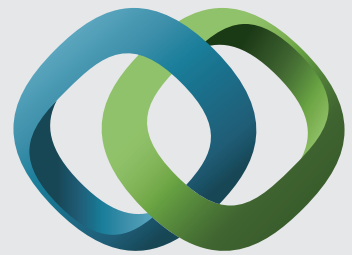

\section{Hindawi}

Submit your manuscripts at

http://www.hindawi.com
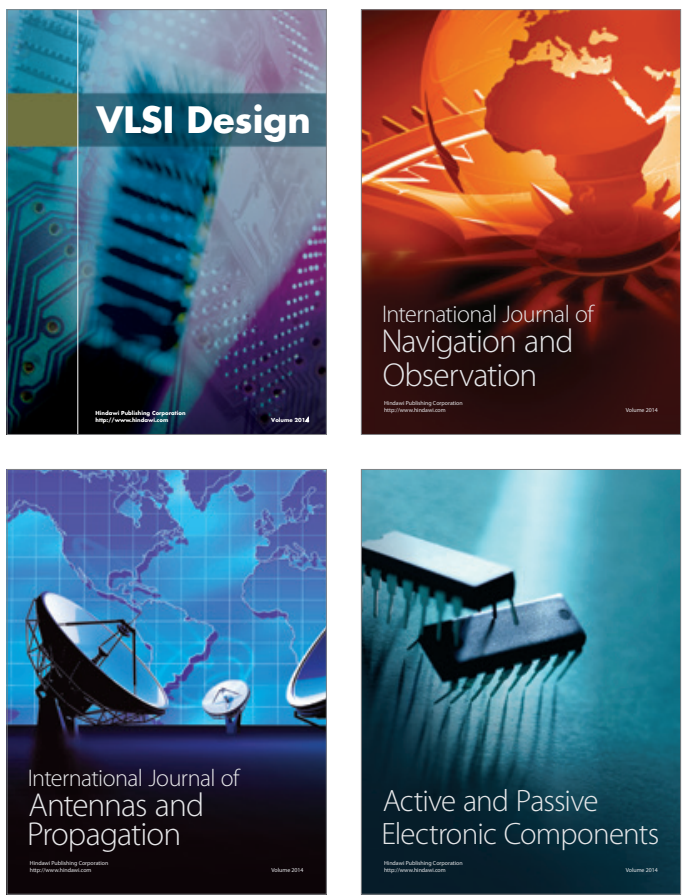
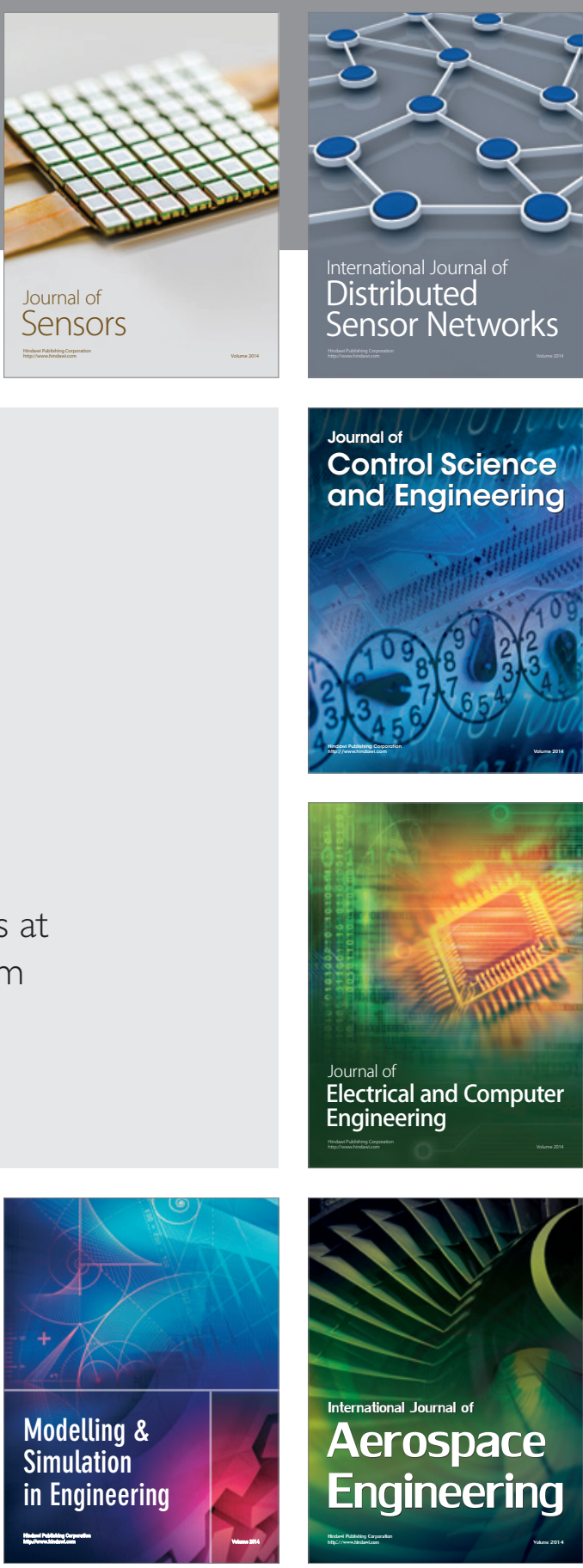

International Journal of

Distributed

Sensor Networks

Journal of

Control Science

and Engineering
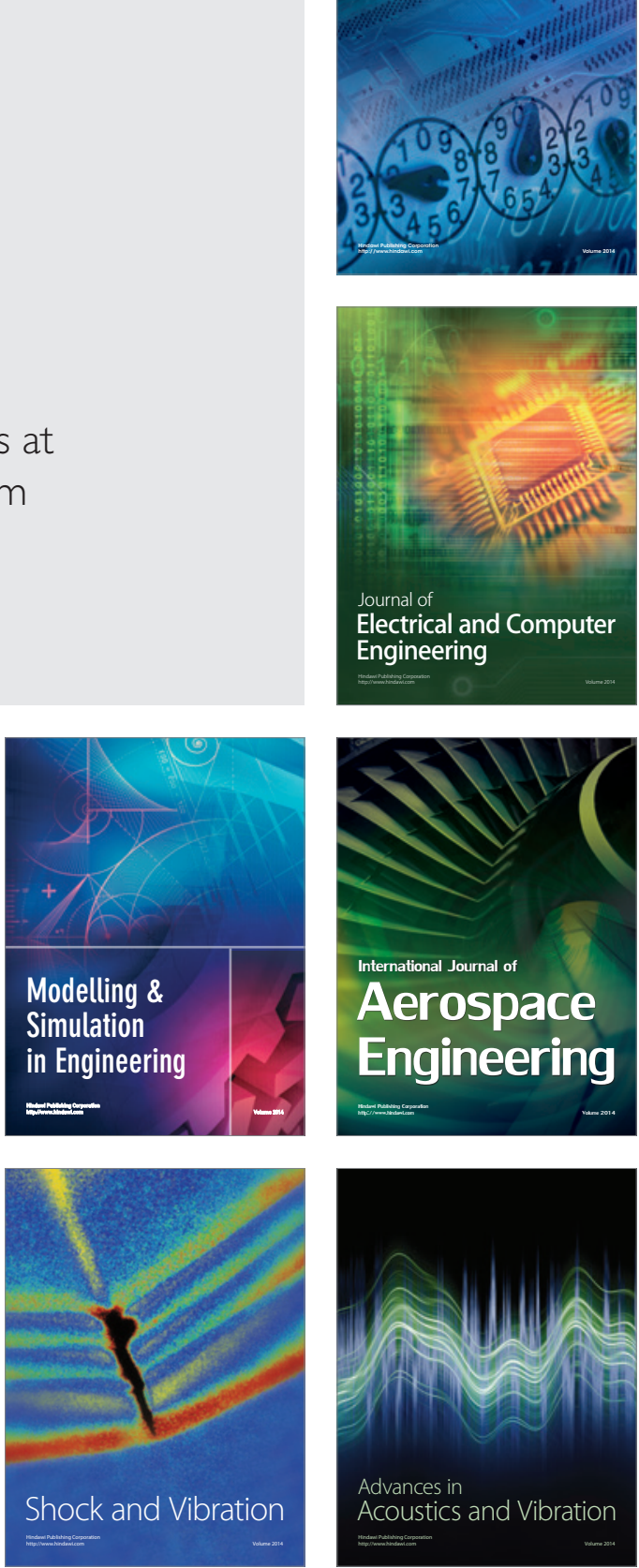